\title{
Modelling and simulation of the tongue during laryngoscopy
}

\author{
M.A.F. Rodrigues ${ }^{\mathrm{a}, 1}$, D.F. Gillies ${ }^{\mathrm{a}}$, P. Charters ${ }^{\mathrm{b}, 2}$ \\ a Department of Computing, Imperial College of Science Technology and Medicine, 180 Queen's Gate, London SW7 2BZ, UK \\ ${ }^{\mathrm{b}}$ Department of Anaesthesia, Walton Hospital, Rice Lane, Liverpool L9 1AR, UK
}

Accepted 19 June 1998

\begin{abstract}
The main aim of our work is to create a realistic model of the behaviour of the upper airways during laryngoscopy. In this procedure the anaesthetist uses a rigid blade to displace the tongue and then inserts a tube into the larynx. The model will serve as the basis of virtual systems which allow anaesthetists to visualise the likely difficulties in carrying out a laryngoscopy, and predict difficult cases in advance. It will also prove useful in building a realistic computer based training system in which anaesthetists interact with simulated tissues. Web-based technology can be used to set the model parameters and run the simulation on a remote machine, while visualising results on a low cost local machine. The main property to be modelled is the behaviour of the tongue as it is mechanically displaced and compressed by the laryngoscope blade. To achieve this, we have used the finite element method in an initial study. The work shows that plausible behaviour can be simulated, and that the elastic properties of the tongue and associated ligaments are significant factors in the procedure. (c) 1998 Elsevier Science B.V. All rights reserved.
\end{abstract}

Keywords: Modelling; Simulation; Tongue; Laryngoscopy

\section{Introduction}

Laryngoscopy is a procedure, carried out by anaesthetists, in which the tongue is mechanically displaced to one side of the mouth using a rigid blade (see Fig. 1). A tube is subsequently inserted into the larynx to feed a mixture of oxygen and anaesthetic gas to the lungs. The procedure is not without difficulty or danger. Consequently we are

\footnotetext{
${ }^{1}$ Corresponding author. Email: mafr@doc.ic.ac.uk. Supported by The National Council for Scientific and Technological Development of Brazil (CNPq) under grant No 200932/95-0.

${ }^{2}$ Supported by a grant awarded by Aintree NHS Trust R\&D Committee.
}

aiming to build a computer model of the process which can be used for prediction of difficult cases and for the development of virtual interactive training devices.

A central issue in building such a model is to find a way to represent the physical behaviour of the tongue so that the relationships between different tongue shapes, blade models and mouth measurements can be identified. This includes modelling of the tongue tissue and its ligaments, the response of the tissue to the instrument used, and the manner in which it changes morphologically during laryngoscopy. Libraries of objects, such as patients and blade types, can then be created, and used to provide the component parts for a variety of virtual medical environments that may be shared, simulated, anal- 


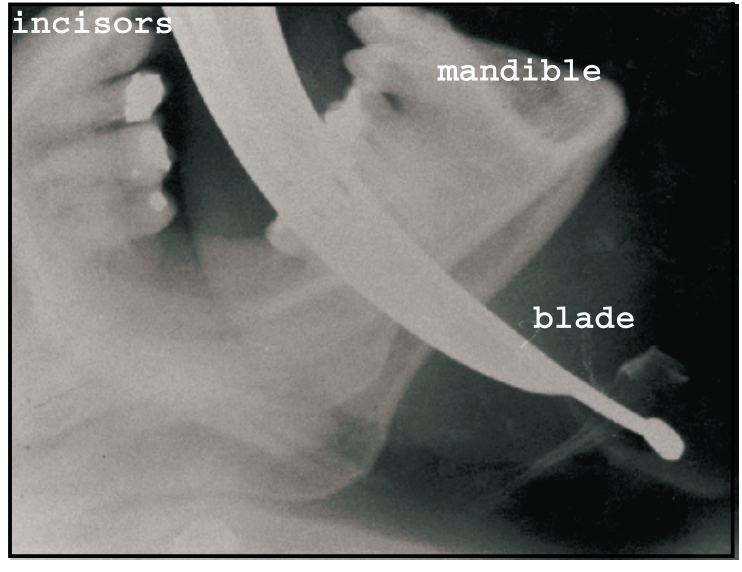

Fig. 1. Lateral radiography of one subject during laryngoscopy.

ysed and visualized by the virtual world of trainee and instructor.

Models of the human tongue have already been proposed in the context of speech production, where the pioneers were Perkell [17] and Kiritani et al. [12]. In Stone et al. [23], the tongue is modelled in 5 segments in the lengthwise and crosswise planes, while Pelachaud et al. [16] retain only 3 segments in these planes. The latter consists of a kinematic model composed of a mesh of 9 three-dimensional triangles that represent charge centres for an equi-potential surface field. In most facial animation systems tongue movement is omitted or is over simplified as a parallelepiped with rudimentary movements $[14,6]$. These models simulate variations of the tongue shapes in response to deliberate movements. By contrast, as laryngoscopy is normally carried out under general anaesthesia, we need to simulate involuntary tongue deformations caused by blade contact. These deformations are difficult to predict because they depend on physical parameters such as elastic tongue properties and contact forces.

Our current model represents the tongue as a uniform elastic resting body which has attachments to the bony structures of the head. To simulate tongue behaviour, a biomechanical model has been investigated. As the tongue is a highly muscular structure, the Finite Element Method serves as a vehicle for modelling the geometric data. The Finite Element Method is a well established technique in the field and its great strength is its versatility. With the condition that satisfactory force-displacement relationships can be established at the element level, there is virtually no limit to the type of structure that can be analysed [8].

The Finite Element Method has also been applied to other biomechanical based simulations. Examples include a surgical simulator in which a three-dimensional computer model of the eye and its surroundings is modelled [22]. Systems for computer-aided plastic surgery are also good examples [24,18,13]. Work has also been done on simulating laparoscopy in real time [7]. The work of Chen [5] in particular, is a novel finite element model of skeletal muscle that can be used both to simulate muscle forces and to visualise the dynamics of muscle contraction. However, experience drawn from the clinical setting and the post-mortem room demonstrates that the tongue differs substantially from normal skeletal muscle.

We assume that the tissue of the tongue has linear isotropic elastic properties, and constant volume. The model consists of a discrete approximation to the geometry measured from lateral MR studies. It is composed of a mesh of nodes and elements in which the functional parts are the structure (nodes, elements, boundary conditions), a set of actions (loads), and a set of responses (nodal displacements). The states of the nodes determine the state of the whole model. Elements represent the material of the structure of the tongue. The geometry of the muscle space plays an important part in the finite element model. It was therefore necessary to take into account the musculature of the tongue. A well known study [15] was used to provide systematic and fairly detailed information concerning the characteristics of each tongue muscle. The geometry of an element was determined by the nodes it is connected to, following the main distribution of the fibers of the tongue. An action changes the state of the structure in terms of its displacements and reactions.

Using standard procedures in the Finite Element Method a set of forces at the vertexes of the data mesh is applied to represent the effects of the elastic stresses (caused by the passive elastic reaction to the outside forces or by active contraction of the internal muscles). The particular shape of the tongue deformation is a function of both the internal stresses and strains within the elastic object and the external forces applied to it. By considering all such forces 
that are related to each nodal point of the tongue, a set of linear static equations for deformation is set up and the Finite Element Method is then simulated forwards and the mesh automatically deforms in response. These equations are essentially based on tongue elasticity properties and blade contact points which are directly related to displacements of the pharyngeal walls during laryngoscopy.

\section{The biomechanics of laryngoscopy}

The normal procedure for laryngoscopy is to insert a metal blade into the right side of the mouth, and then attempt to displace the tongue to the left side keeping the blade in the mid-line as much as possible [2] (see Fig. 2). However, this procedure is not simple and sometimes the tongue must also be pushed down by the blade in order to expose the vocal cords and larynx of the patient.

In the ideal case, the forces applied by the anaesthetist to the laryngoscope should be directed parallel to the axis of the handle [19]. This is shown as force $F_{b}$ in Fig. 3. According to Newton's first law, for a body in equilibrium the sum of all force vectors acting on the body must be zero and the sum of the moments of all forces around any axis must also be zero. If the blade touches the maxillary incisors, the direction of forces will be opposite to those on the tongue [3]. Furthermore, the point of contact of the former will be closer to the handle than the latter (see Fig. 3). When these two opposing forces act along the axis of the handle, we will consider their sum. In the case where the blade does not touch the incisors, the resulting forces transmitted by the tongue onto the blade $F_{t}$ have different points of applica-

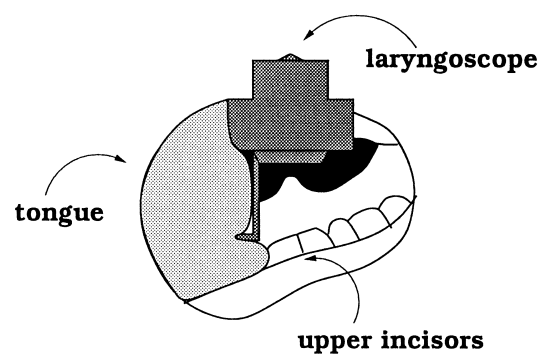

Fig. 2. Schematic positioning of laryngoscope blade and tongue relative to teeth during laryngoscopy.

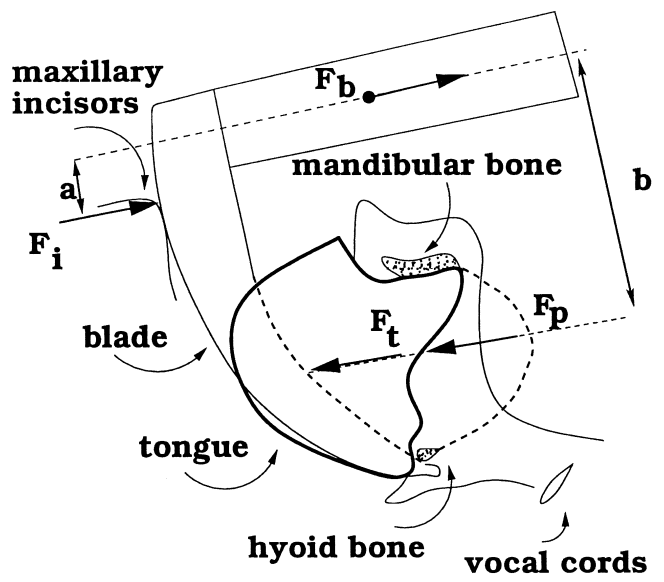

Fig. 3. Schematic representation of forces acting during laryngoscopy and the "peardrop" effect caused by tongue deformation.

tion, magnitude and direction and produce a moment onto the laryngoscope $F_{t} b$. To prevent the laryngoscope from turning, this moment should be balanced by a counter-acting moment exerted by the hand of the anaesthetist.

When two solid bodies collide, the material around the points of contact must deform to prevent their interpenetration. The deformation gives rise to a contact force that tends to push the two bodies away from each other. The force disappears if and when the two bodies move apart. The forces and moments acting upon tongue, incisors, pharyngeal walls and blade are described by the following equations:

$$
\begin{aligned}
F_{b}+F_{i} & =F_{t}+F_{p} \\
M_{b}+M_{i} & =M_{t}+M_{p}
\end{aligned}
$$

where, $F_{b}$ is the force applied by the anaesthetist to the blade, $F_{i}$ is the force applied to the blade by the maxillary incisors teeth, $F_{t}$ is the force applied to the blade by the tongue, $F_{p}$ is the force applied to the tongue by the pharyngeal walls, $M_{b}$ is the moment exerted by the anaesthetist on the blade, $M_{i}$ is the moment exerted by force of the maxillary incisors, $M_{t}$ is the moment exerted by the tongue, $M_{p}$ is the moment exerted by the pharyngeal walls, and, $a$ and $b$ are the distances from the mid-point of the handle of blade to the application point of $F_{i}$ and $F_{t}$, respectively. 
The above equations can be rewritten as follows to obtain the equations for $F_{i}$ and $F_{t}$, according to the following cases:

- Case I: The blade does not touch incisors $\left(F_{i}=\right.$ $\left.0, M_{i}=0\right)$

$$
\begin{gathered}
F_{b}=F_{t}+F_{p} \\
M_{b}=M_{t}+M_{p}
\end{gathered}
$$

therefore,

$$
F_{t}=\frac{M_{b}-F_{p} b}{b}
$$

- Case II: The blade touches incisors

$$
\begin{gathered}
F_{b}+F_{i}=F_{t}+F_{p} \\
M_{b}+M_{i}=M_{t}+M_{p}
\end{gathered}
$$

therefore,

$$
\begin{gathered}
F_{i}=\frac{M_{b}-F_{b} b}{b-a} \\
F_{t}=\frac{M_{b}+F_{i} a-F_{p} b}{b}
\end{gathered}
$$

It has been observed that force and torque may vary significantly between experienced laryngoscopists [10]. This is because force and torque depend on the anaesthetist's technique and skill varies significantly even among experienced laryngoscopists. Increased force on the tongue will cause an increase in compression which will depend directly on the elastic properties of its different muscles and ligaments.

Obviously, a realistic three-dimensional model of laryngoscopy will not be simple given the complicated tongue geometry and behaviour. As an intermediate step we first investigated a two-dimensional model to gain insight into the behaviour of soft tissue. Though this is a simplification of what actually happens, under simplified boundary constraints, it represents the first significant step towards a three-dimensional model of this difficult medical application. Two-dimensional modelling has already had important real world implications in suggesting a previously unrecognised causative mechanism for difficult intubation called - the "hi-slung" mandible [4].

\section{A finite element model of the tongue}

Muscles change shape due to the dynamic interactions of contraction and contact forces [11]. Deformation of the tongue will be caused by the blade forces, and the reactive forces of the pharyngeal walls resulting in the "peardrop" appearance (as shown in Fig. 3). It is necessary to model the ways in which the above mentioned tissues interact with each other. In order to simulate the interaction of the tongue with the soft tissue around the base of the mandible, constraints, expressed as springs forces exerted on the surface of tongue, are defined.

Following a classical approach, the boundary conditions of our problem are formed by the input forces applied to the surface of the model and a set of points that remain fixed, or at least, have restricted movement. More specifically, the primary boundary conditions are the forces acting on the tongue (essentially applied at the dorsum of the tongue) and the rigid structures to which the tongue is attached (see Fig. 4). These rigid structures are defined by (1) the nodal points at the front edge of the mandible, (2) a nodal point at the back end on the basal plane of the tongue which is directly fixed to the hyoid bone, and (3) the styloglossus muscle and stylohyoid ligaments which extend from the base of skull to the dorsum of the tongue and to the direction of hyoid bone, respectively. During laryngoscopy, further movement of hyoid bone is limited by the stylohyoid ligaments. Different degrees of calcification in the ligaments have been identified as a cause of difficult intuba-

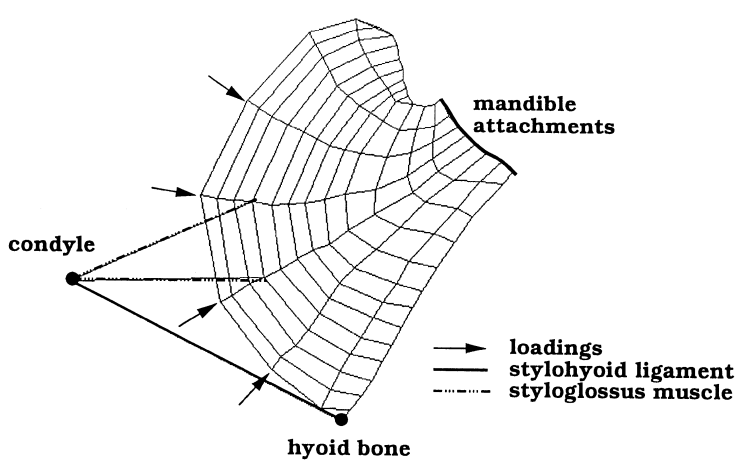

Fig. 4. Schematic boundary conditions of the finite element of the tongue. 
tion. The extension range of the stylohyoid ligaments when mouth is opened and the laryngoscope inserted, was estimated from MR images and X-Ray studies.

Given the loading forces imposed at a node (or a set of nodes) on the boundary we can deduce the displacement field. The consistency of the boundary conditions in terms of the resulting displacements was examined by the anaesthetists and compared with results from X-Ray records, and the computation repeated with new parameters until convergent results were obtained.

\subsection{Musculature of the tongue}

The tongue is modelled as an elastic body composed of finite elements. The element's deformation is restricted to affine transformations of their rest shapes. The lingual muscles are anatomically divided into two groups, the extrinsic and the intrinsic. The former is characterized by the fact that the muscles all originate outside the tongue mass, having attachments to the bony structures of the head. In contrast, the latter has no such bony attachments, and the muscles rise from and end up within the bulk of the tongue [9]. In this study the tongue is defined as an area that contains three extrinsic lingual muscles (genioglossus, hyoglossus and styloglossus) and four intrinsic muscles (superior and inferior longitudinalis, transversus and verticalis). The rigid structures to which the above mentioned muscles attach are the mandible, the hyoid bone, the styloid processes and the mastoid processes.

\subsection{Structural analysis}

Depending upon the nature of the loading, the structure of the tongue may respond in a number of ways. For example, it may deform statically in a stable elastic manner. The purpose of structural analysis is to predict accurately the response of a given structure to a given loading, i.e. to calculate the distribution of internal forces and displacements or to estimate the level of loading at which instability occurs under the prescribed conditions of geometry and loading.

The choice of the mesh to represent the tongue is not necessarily unique and the idealization, shown in Fig. 4, is only one of an infinite number of possibili- ties that could be employed. Naturally, the geometry of the actual structure needs to be matched as closely as possible, and the expected response of its structure to the applied loading must also be considered in deciding the detailed distribution of elements. This latter point is important, since there will perhaps be regions of the structure where displacements, strains, and stresses vary in a complicated manner and other regions where the variation is of a simple nature.

If the element properties are properly formulated, then in general the more elements that are used in representing a structure, the better will be the accuracy of the results. When it has been decided how many elements are going to be used for a particular finite element analysis of a continuum structure it still has to be decided how these elements should be arranged. In deciding on the arrangement of elements there should, as far as is possible, be taken into account the expected behaviour of the structure under load so that rather more elements are placed in regions of anticipated complicated structural behaviour and rather less elements in regions where simple response is expected [1].

The model that we are using can be thought of as a set of beam elements subjected to loads. For a linear, homogeneous, isotropic material, the mechanical material properties of the beam are represented by the Young's modulus, E, and by the Shear modulus, G. In view of the lack of concrete data for the elastic parameters of the tongue we have followed the approach of Chen [5] who found that a good initial approximation can be taken from the properties of rubber materials.

The tongue was built from 214 straight beam elements, all with the same properties, each 9.906 $\mathrm{mm}$ long and with $1.9812 \mathrm{~mm}$ constant square cross section. The determination of the moment of inertia was done by direct integration, as shown in Eq. (6).

$I_{x}=I_{y}=\frac{b h^{3}}{12}=1.248 \mathrm{~mm}^{4}$

The polar moment of inertia and the area of inertias are related by the following equation:

$J=I_{x}+I_{y}=2.496 \mathrm{~mm}^{4}$

The tongue control parameters are (1) location on bone attachments and ligaments, (2) contact regions, (3) type of deformation (linear, non-linear), (4) phys- 
ical properties, and (5) loadings points. The Finite Element Method assumes that displacements under load are small and proportional to the applied load, which leads to a set of constitutive linear equations.

The tongue is fully restrained at the front part of the mandible to which it is attached and at the nodal point at the back end on the basal plane (hyoid bone), which is presently constrained so that only displacements in the horizontal plane are allowed. Each node is identified in global coordinates, for all six degrees of freedom. In particular, all nodes are prevented from having translations and rotations in the $z$ plane. The mesh is loaded with non-linear forces whose magnitudes were taken from forcemeasuring experiments during normal laryngoscopy [3].

The mechanical behaviour of the tongue, particularly the elasticity of the soft tissues, is one of the primary determinants of the change of its appearance during the insertion of the blade. The amount of displacement of a particular point is determined by its distance from the point being moved, the elastic- ity of surrounding tissue, and the influence of boundary conditions. The elasticity range of the soft tissue, represented mathematically in this work by Young's modulus $(E)$ and by Shear modulus $(G)$, varies between 0.0007 to $0.004 \mathrm{GPa}$ and 0.0002 to 0.001 $\mathrm{GPa}$ [25], respectively. In a more detailed model, these parameters may need to depend on their orientation with respect to the underlying orientation of fibers of the tissue.

\section{Results}

In this study, the blade shapes shown in Figs. 5 and 6 are initially positioned forward from the upper incisors and constrained by the size and elastic properties of the tongue and its ligaments. The mouth measurements were extracted from X-Rays and are represented by the bony points: I, the tip of upper incisors; $\mathrm{T}$, the hyoid point above the larynx; J, the mid-point between the mandibular condyles and $\mathrm{S}$, the internal mid-point on the front of the mandible.
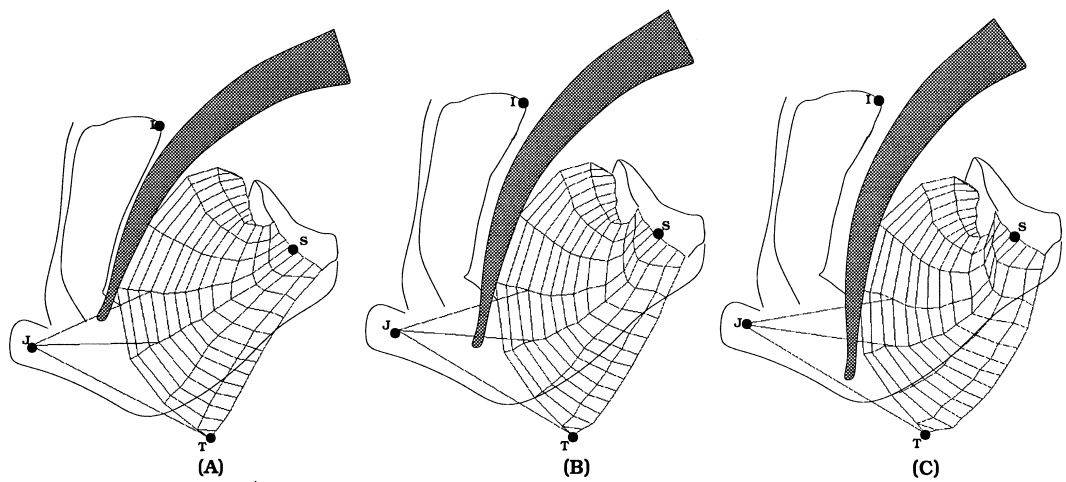

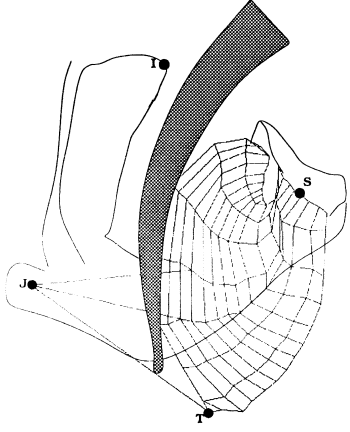

(D)

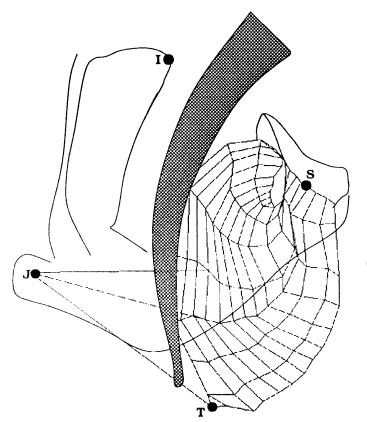

(E)

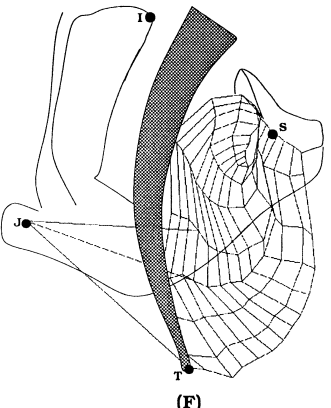

(F)

Fig. 5. Finite element of tongue deformation during an easy case of laryngoscopy. 

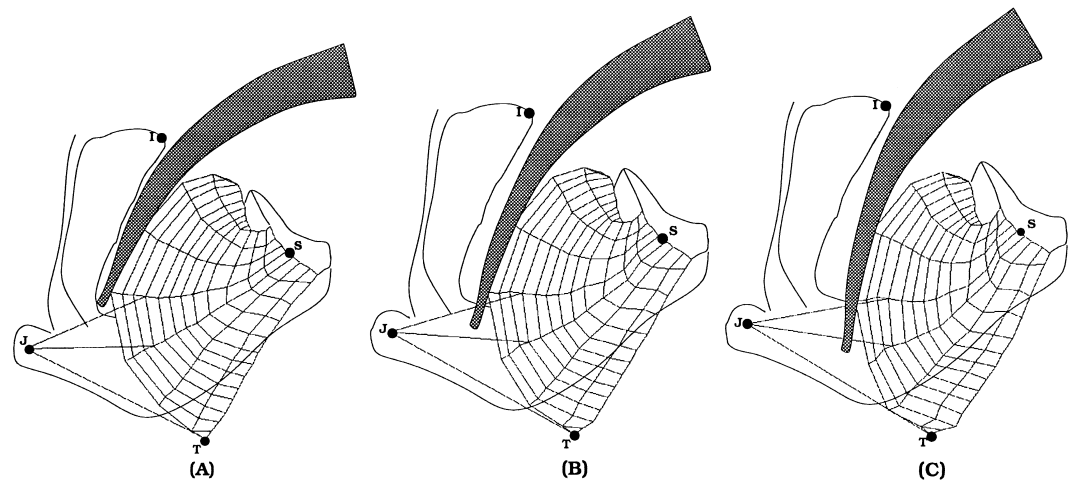

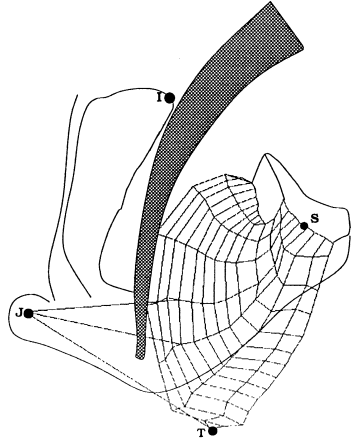

(D)

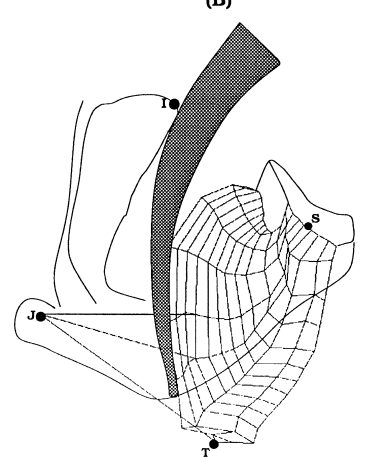

(E)

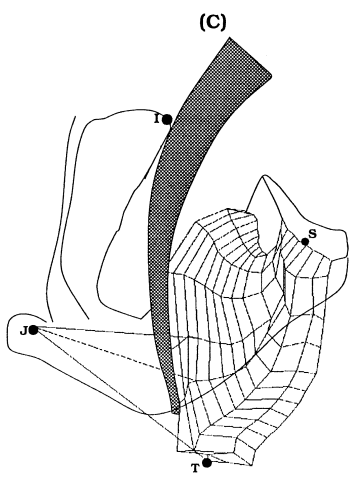

(F)

Fig. 6. Finite element of tongue deformation during a difficult case of laryngoscopy.

In Fig. 5, the blade tip can be made to touch the hyoid and the tongue becomes maximally compressed causing the "peardrop" appearance. The size and elasticity of the tongue, and the degree of calcification of its main ligaments, defined in this first finite element model, indicate that the subject will not cause a difficult laryngoscopy. However, increasing the stiffness of the elements we get the result shown in Fig. 6. The blade does come in contact with the upper incisors and is unable to contact the hyoid point, thus indicating failed laryngoscopy. The line of view to the laryngeal inlet is poor and the larynx cannot be seen at all. Even using low stiffness values for its ligaments, the elastic behaviour of the tongue still prevents the blade from contacting the hyoid point. If a bigger tongue size had been used there would have been a greater likelihood of predicting failed intubation.

The results demonstrate that difficulty in intubation does depend on the elastic properties of the tongue. Thus, the physical modelling provides an improvement over our earlier work using osseous factors $[4,20]$ and eyeline deviation [21] where the same data indicated easy intubation.

\section{Conclusions}

The focus of this work was to investigate the extent to which a finite element model can be used to (1) approximate the elastic behaviour of the tongue and its main ligaments when subjected to loads during the blade contact, (2) derive the equilibrium equations from both the shape and the material properties of its elastic tissues, and (3) define a realistic deformation as the tongue changes shape in response to external forces.

This study showed that, besides analysis of mouth measurements and blade eyeline deviation, further factors should be considered when attempting to predict difficult intubation. In particular, it was observed that the size and elastic behaviour of the tongue, as well the degree of calcification in its ligaments, may have a great influence on the laryn- 
goscopy outcome. The subject studied in this work would not be considered an easy case if elasticity properties of the tongue were unfavorable, such as those used in the simulation shown in Fig. 6.

In view of the complexity of the geometric and functional structure of the tongue during laryngoscopy, the finite element study proposed in this work will serve as a stepping stone for future research in the area, such as exploring different formulations of the tongue model to achieve more realistic behaviour. It will involve three-dimensional contact analysis modelling, including surface friction effects between blade and tongue.

In the short term, the hope is that if the three-dimensional forces are simulated correctly, then the correct changes in the three-dimensional muscle shape of the tongue will be automatically produced and propagated to the surface. The use of a three-dimensional force-measuring laryngoscope to collect input data for the three-dimensional model is under way and will be useful in finding out factors causing difficulty.

Validation is an important part of any control strategy, and presently it is done by subjective inspection. In the long term, correlation with real laryngoscopies and the use of simulation systems will help to improve the validation and improve the realism of the model. We anticipate that the validation of the three-dimensional model will relate to predictions derived from the two-dimensional analysis.

In the future, it is intended that an electro-mechanical interface will allow anaesthetists to feel contact forces from instruments as they interact with threedimensional simulated tissues. This virtual environment may be developed using recent Web-based technologies. Users of such a system would, at different locations, set the model parameters and run the simulation on a remote machine, and then visualize and interpret the results locally.

\section{References}

[1] Klauss-Jurgen Bathe, Finite Element Procedures in Engineering Analysis (Prentice-Hall, 1982).

[2] M.J.L. Bucx, P.A.E. Scheck, R.T.M. Geel, A.H.den Ouden, R. Niesing, Measurement of forces during laryngoscopy, Anaesthesia 47 (1992) 348-351.
[3] M.J.L. Bucx, C.J. Snijders, R. Geel, C. Robers, H. Giessen, W. Erdmann, T. Stijnen, Forces Acting on the Maxillary Incisor Teeth during Laryngoscopy using the Macintosh Laryngoscope, Anaesthesia 49 (1994) 1064-1070.

[4] P. Charters, Analysis of Mathematical Model for Osseous Factors in Difficult Intubation, Canadian Journal of Anaesthesia 41 (7) (1994) 594-602.

[5] D.T. Chen, Pump It Up: Computer Animation of a Biomechanically Based Model of Muscle using the Finite Element Method (PhD thesis, Massachusetts Institute of Technology, October, 1991).

[6] M.M. Cohen, D.W. Massaro, Modelling coarticulation in synthetic visual speech, in: D. Thalmann, N. Magnenat-Thalmann, eds., Computer Animation'93 (Springer-Verlag, 1993).

[7] S. Cotin, H. Delingette, J.M. Clement, V. Tassetti, J. Marescaux, N. Ayache, Volumetric Deformable Models for Simulation of Laparoscopic Surgery, Computer Assisted Radiology (1996) 793-798.

[8] D.J. Dawe, Matrix and Finite Element Displacement Analysis of Structures (Oxford University Press, New York, 1984).

[9] H. Gray, Gray's Anatomy: The Anatomical Basis of Medicine and Surgery (38th British, Bannister, Lawrence H. and Berry, Martin M., Churchill Livingstone, 1995).

[10] R.H. Hastings, E.D. Hon, C. Nghiem, Force and Torque vary between Laryngoscopists and Laryngoscope Blades, Anaesth. Analg. 82 (1996) 462-468.

[11] N. Kikuchi, J.T. Oden, Contact Problems in Elasticity Study of Variational Inequalities and Finite Element Methods (Philadelphia, Society for Industrial \&Applied Mathematics, 1988).

[12] S. Kiritani, K. Miyawaki, O. Fujimura, J.E. Miller, A Computational Model of the Tongue, Ann. Bull. RILP 10 (1976) 243-251.

[13] R.M. Koch, M.H. Gross, F.R. Carls, D.F. von Buren, G. Fankhauser, Y.I.H. Parish, Simulating Facial Surgery Using Finite Element Models, Computer Graphics Proceedings of SIGGRAPH'96 (New Orleans, 1996) 421-428.

[14] N. Magnenat-Thalmann, D. Thalmann, The direction of synthetic actors in the film Rendez-vous à Montréal, IEEE Computer Graphics and Applications (1987) 9-19.

[15] K. Miyawaki, A Study on the Musculature of the Human Tongue, Annual Bulletin (Research Institute of Logopedics \&Phoniatrics) 8 (1974) 23-50.

[16] C. Pelachaud, C.M.A. M Overveld, Modelling and Animating the Human Tongue during Speech Production, IEEE Computer Animation'94 (1994) 40-49.

[17] J.S. Perkell, A Physiologically-Oriented Model of Tongue Activity in Speech Production (PhD thesis, Massachusetts Institute of Technology, 1974).

[18] S. Pieper, J. Rosen, D. Zeltzer, Interactive Graphics for Plastic Surgery: A Task-Level Analysis and Implementation, Symposium on Interactive 3D Graphics (Cambridge, Massachusetts, 1992) 127-134.

[19] J.T. Roberts, Fundamentals of Tracheal Intubation (Grune \&Stratton, 1983).

[20] M.A.F. Rodrigues, D.F. Gillies, P. Charters, R. Marks, Computer Assisted Pre-operative Laryngoscopy, Compugraphics'96 (1996) 114-120. 
[21] M.A.F. Rodrigues, D.F. Gillies, P. Charters, R. Marks, Development of a Computer System for Prediction of Difficult Laryngoscopy, Abstract in the British Journal of Anaesthesia 78 (4) (1997) 466-467.

[22] M.A. Sagar, D. Bullivant, G.D. Mallison, P.J. Hunter, I. Hunter, A virtual environment and model of the eye for surgical simulation, Computer Graphics Proceedings of SIGGRAPH'94 (Orlando, 1994) 205-212.

[23] M. Stone, Toward a Model of the Three-Dimensional Tongue Movement, Journal of Phonetics 19 (1991) 309-320.

[24] D. Terzopoulos, K. Waters, Physically-based facial modelling analysis and animation, Journal of Visualization and Computer Animation 1 (2) (1990) 73-80.

[25] S.P. Timoshenko, J.M. Gere, Mechanics of Materials (PWS-KENT Publishing Company, Boston, 1990).

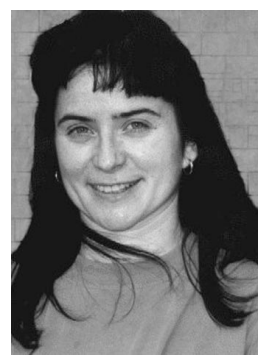

M. Andréia Formico Rodrigues received the B.S. degree in Computational and Applied Mathematics from UNICAMP (State University of Campinas), São Paulo, Brazil, in 1989. She then obtained the M.Sc. degree in Computer Graphics from the School of Electrical Engineering at the same university. She is currently completing a Ph.D. in the Department of Computing at Imperial College, London. Her main research interests are in mathematical modelling, computer graphics, animation, and simulation.

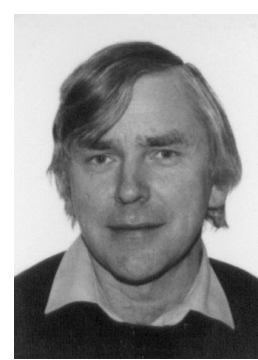

Duncan Gillies graduated from Cambridge University with a degree in Engineering Science in 1971. He subsequently obtained the M.Sc. degree in Computing and a Ph.D. in the area of artificial intelligence from London University. After teaching for six years at the Polytechnic of the South Bank, he moved to the Department of Computing in Imperial College where he is now a Reader. His research interests are in graphics and computer vision.

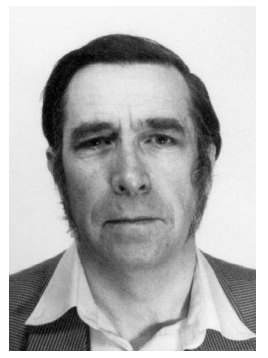

Peter Charters is a Consultant in Anaesthesia and Intensive Care with special interests in mathematical models of clinical problems especially the upper airway and difficult intubation. He is also interested in hyperbaric intensive care medicine. In addition to higher training in anaesthesia and general medicine he has obtained a degree in Pure and Applied Mathematics from the Open University. He has published several articles mainly relate to clinical studies of the upper airway. 